\title{
Pneumocephalus during labor analgesia using the combined spinal-epidural technique
}

\author{
Hyun-Young Lim ${ }^{1}$, Ji-Won Choi ${ }^{2}$, Ae Ryoung Lee ${ }^{1}$, Hyo Won Park ${ }^{1}$, and Duck Hwan Choi ${ }^{1}$ \\ Department of Anesthesiology and Pain Medicine, 'Samsung Medical Center, Sungkyunkwan University School of Medicine, \\ ${ }^{2}$ Daejin Medical Center, Seoul, Korea
}

\begin{abstract}
Although neuraxial labor analgesia has been considered very safe, it may result in some complications related to the procedural techniques. In rare cases, the loss of resistance to air (LORA) technique, a popular method for identifying the epidural space, may be associated with intracranial air. We report a case of pneumocephalus featuring sudden-onset headache after the combined spinal epidural (CSE) technique for labor analgesia.
\end{abstract}

A 31-year-old, full-term primigravida requested epidural analgesia in labor. Her body weight was $52 \mathrm{~kg}$, and she was 157 $\mathrm{cm}$ tall (body mass index $=21.1 \mathrm{~kg} / \mathrm{m}^{2}$ ). She had no particular medical history. It had been an uneventful pregnancy and there were no contraindications to regional analgesia. In the right lateral decubitus position, a lumbar puncture was performed with a $25 \mathrm{G}$ Whitacre spinal needle, at the L4-5 interspace as part of the cerebrospinal fluid (CSF) technique. After free aspiration of the CSF was assured, $0.2 \%$ ropivacaine and fentanyl $20 \mu \mathrm{g}$ were injected intrathecally. Then, LORA was attempted with a $17 \mathrm{G}$ Tuohy needle (Arrow International, Inc., Reading, PA, USA) via the median approach by a trainee anesthesiologist under supervision. Following the failure of the trainee to place the epidural catheter, it was inserted by the attending anesthesiologist. About 2 hours after the epidural insertion, the patient's labor pain reappeared and worsened, and the decision was made to remove the catheter and retry the insertion. After giving an intrathecal dose (same as the above), a senior trainee retried the epidural catheter insertion, and the catheter was placed clearly and uneventfully at the L3-4 interspace, using LORA. The amount of air used to identify the epidural space was about $8 \mathrm{ml}$. After about
5 minutes, the patient complained of moderate headache combined with nuchal discomfort. One hour after the procedure, she began to complain of severe headache. As this headache had different features from post-dural puncture headache (PDPH), it was not thought to be PDPH. Because of the continued severe headache and the possibility of catheter malpositioning, the catheter was removed.

After 4 more hours of labor, during which mild labor pain reappeared, the patient had a normal vaginal delivery of a male infant with Apgar scores of 9 at $1 \mathrm{~min}$ and 10 at $5 \mathrm{~min}$. The following day, as she continued to report severe headache, apparently not postural, a cranial computed tomography (CT) scan was done following a neurology consultation. The scan showed large amounts of intracranial air in the subarachnoid space (Fig. 1A). The patient was given oral analgesics for pain control and $100 \%$ oxygen via a facial mask to reduce the size of the air bubbles. She had no further complaints and was discharged after 4 days without any specific complaints regarding headache or neurologic symptoms. After 1 week, a repeat cranial CT scan showed complete absorption of the air bubbles (Fig. 1B).

Headache after epidural anesthesia is generally ascribed to unintentional dural puncture with consequent CSF leakage through the hole in the dura resulting in low CSF pressure [1]. This commonly seen headache, PDPH, usually becomes prominent 24-48 hours after the dural puncture. The typical symptom is moderate to severe postural headache, which is aggravated on standing or sitting. When the headache interferes with a patient's daily life, an epidural blood patch is commonly used as a definite

Corresponding author: Duck Hwan Choi, M.D., Department of Anesthesiology and Pain Medicine, Samsung Medical Center, Sungkyunkwan University School of Medicine, 50, Ilwon-dong, Gangnam-gu, Seoul 135-710, Korea. Tel: 82-2-3410-0369, Fax: 82-2-3410-0361, E-mail: duckhwanc@gmail.com

() This is an open-access article distributed under the terms of the Creative Commons Attribution Non-Commercial License (http:// creativecommons.org/licenses/by-nc/3.0/), which permits unrestricted non-commercial use, distribution, and reproduction in any medium, provided the original work is properly cited. 

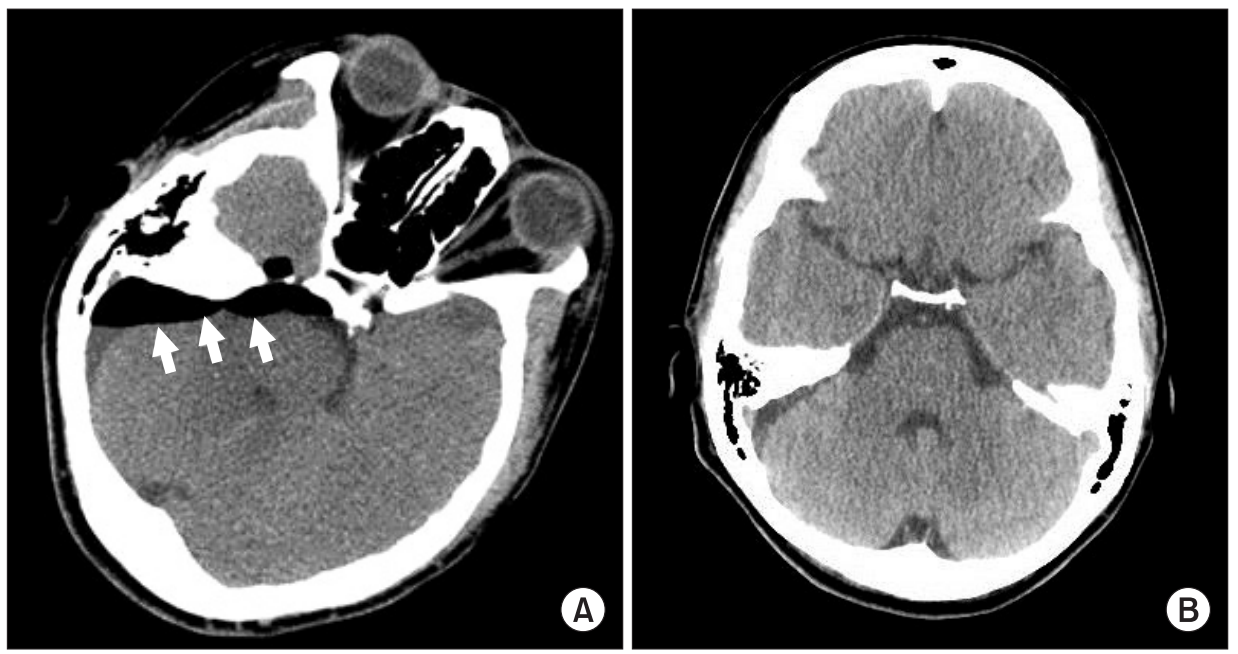

Fig. 1. Computed tomography scan of the brain on postpartum day one, (A) shows large amounts of intracranial air in the subarachnoid space, (B) shows complete resolution of the air bubbles.

treatment. The most common symptom of pneumocephalus is severe headache, having a sudden onset, with gradual improvement over 4-5 days as the air is resorbed [2].

Reynolds and Speedy [3] suggested that in cases of PDPH without CSF leakage, the sequence of events is as follows: the dura mater is punctured by the Tuohy needle, and the delicate arachnoid mater tears, leading later to CSF loss and PDPH. In our case, the patient developed moderate headache about 5 minutes after the second epidural catheter insertion attempt, without CSF leakage. We assumed that air could have been infused into the patient's cranium either through an unrecognized dural puncture or through a dural defect in the first or second attempt and a subsequent arachnoid tear.

Unfortunately, the trainee who performed the epidural analgesia did not consider this headache to be a consequence of the epidural technique because there was no evidence that the dura had been injured. This oversight highlights how important it is that practitioners be familiar with all potential complications of the techniques they use. Furthermore, sufficient and clear explanations must be given to parturients about possible complications, even rare ones, before they undergo this procedure.

There are several ways to avoid pneumocephalus. First, iden- tification of the epidural space by LOR with normal saline may be superior to the use of air. Complications related to the use of saline rarely have been reported. LORA, on the other hand, has been associated with pneumocephalus, nerve root compression, subcutaneous emphysema, venous air embolism, and insufficient analgesia due to unblocked segments caused by epidural air [4]. Second, to prevent pneumocephalus arising from multiple attempts at epidural needle insertion, practitioners can use ultrasonography. Spence et al. [5] provided evidence that preprocedural ultrasonography is a useful adjunct for the placement of epidural catheters in obstetrical patients. In this case, CSE analgesia was performed with difficulty through several trials by many practitioners. If more than a few attempts are required for the procedure, ultrasound guidance may be useful to identify the epidural space. Finally, in order to minimize the possibility of pneumocephalus when performing labor epidural analgesia with LORA, the minimum amount of air should be used. In addition, if headache develops immediately following the epidural procedure, with or without CSF leakage, practitioners should suspect pneumocephalus and immediately initiate conservative therapy. Brain CT should then be performed for confirmation.

\section{References}

1. Han CS, Yu JS, Kim IH, Kim YJ, Kim CS, Ahn KR. Headache and pneumocephalus after lumbar epidural block: A case report. J Korean Pain Soc 1996; 9: 251-5.

2. Nafiu OO, Urquhart JC. Pneumocephalus with headache complicating labour epidural analgesia: should we still be using air? Int J Obstet Anesth 2006; 15: 237-9.

3. Reynolds F, Speedy HM. The subdural space: the third place to go astray. Anaesthesia 1990; 45: 120-3.

4. Kim YJ, Baik HJ, Kim JH, Jun JH. Pneumocephalus developed during epidural anesthesia for combined spinal-epidural anesthesia. Korean J Pain 2009; 22: 163-6.

5. Spence D, Nations R, Rivera O, Bowdoin S, Hazen B, Orgill R, et al. Evidence-based anesthesia: The use of preprocedural ultrasonography during labor to facilitate placement of an epidural catheter. AANA J 2012; 80: 223-30. 